\title{
Multi-focus coherent anti-Stokes Raman scattering microscopy
}

\author{
Mamoru Hashimoto ${ }^{1}$, Tsutomu Araki ${ }^{1}$, and Satoshi Kawata ${ }^{2}$
}

${ }^{1}$ Graduate School of Engineering Science, Osaka University, 1-3 Machikaneyama, Toyonaka, Osaka 560-8531 Japan

${ }^{2}$ Graduate School of Engineering, Osaka University

Recently, new nonlinear microscopy using coherent anti-Stokes Raman scattering (CARS) spectroscopy has been developed, and the non-staining imaging with three-dimensional resolution has been demonstrated[1-3]. Figure 1 shows the CARS process. In CARS spectroscopy, two laser beams with different wavelengths are used for excitation. When the frequency difference $\omega_{1}-\omega_{2}$ is equal to the frequency of the Raman active molecular vibration $\Omega$, CARS is generated as the coherent emission at $2 \omega_{1}-\omega_{2}$. The advantages of CARS spectroscopy over conventional Raman (spontaneous Raman) spectroscopy are the high intensity emission and the separation from undesired fluorescence signal because CARS is the anti-Stokes side emission.

We have developed a multi-focus CARS microscope system for fast multi-spectral imaging. Figure 2 and 3 show the schematic layout of the developed system and the principle of multi-focus CARS microscopy, respectively. The system consists of a picosecond tunable laser based on an optical parametric amplifier excited by a regeneration amplifier, a rotating micro-lens array disk, and a transmission microscope with an intensified CCD camera. A few tens of points are excited simultaneously by the micro-lens array disk, and these excited spots are scanned by rotating the array disk. Therefore, a CARS image is obtained by the intensified CCD at once.

Figure 4 shows CARS images of the mixture of polystyrene (diameter $=4.5 \mu \mathrm{m}$ ) and glass beads (diameter $=3 \sim 5 \mu \mathrm{m}$ ) observed (a) at $990 \mathrm{~cm}^{-1}$ that is near the peak of the phenyl breath mode of polystyrene and (b) at $1050 \mathrm{~cm}^{-1}$ apart form the band peak. One can recognize that some beads with larger diameters in Fig. 4 (a) shows brighter image than those in Fig. 4 (b), though the intensity of the beads with smaller diameters was not changed appreciably. Figure 5 shows resultant CARS spectra of beads positioned at the cross point of $\alpha$ and at $\beta$ in Fig. 4 . The spectral distinction of these beads was clearly detected, and therefore, the distribution of analytical sample can be identified by the multi-spectral CARS images. We have also developed the CARS microscopy system with two synchronized ps Ti:Sapphire lasers have narrower spectral band widths. The results of the new system will be also shown.

Reference

[1] A. Zumbusch, G. R. Holtom, and X. S. Xie, Phy. Rev. Lett . 82, 4142-4145 (1999).

[2] M. Hashimoto and T. Araki, SPIE 3749 , 496-497 (1999).

[3] M. Hashimoto, T. Araki, and S. Kawata, Opt. Lett. 25, 1768-1770 (2000). 


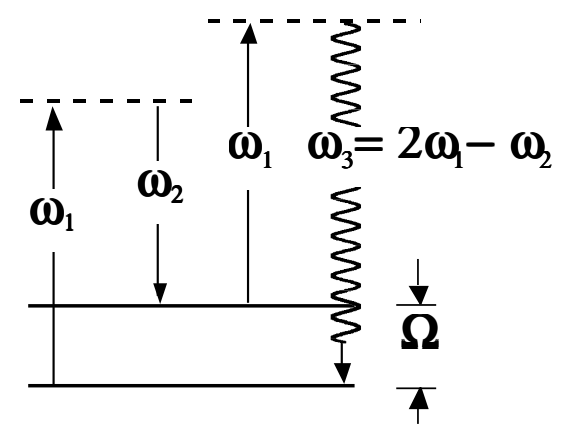

Figure 1. CARS process

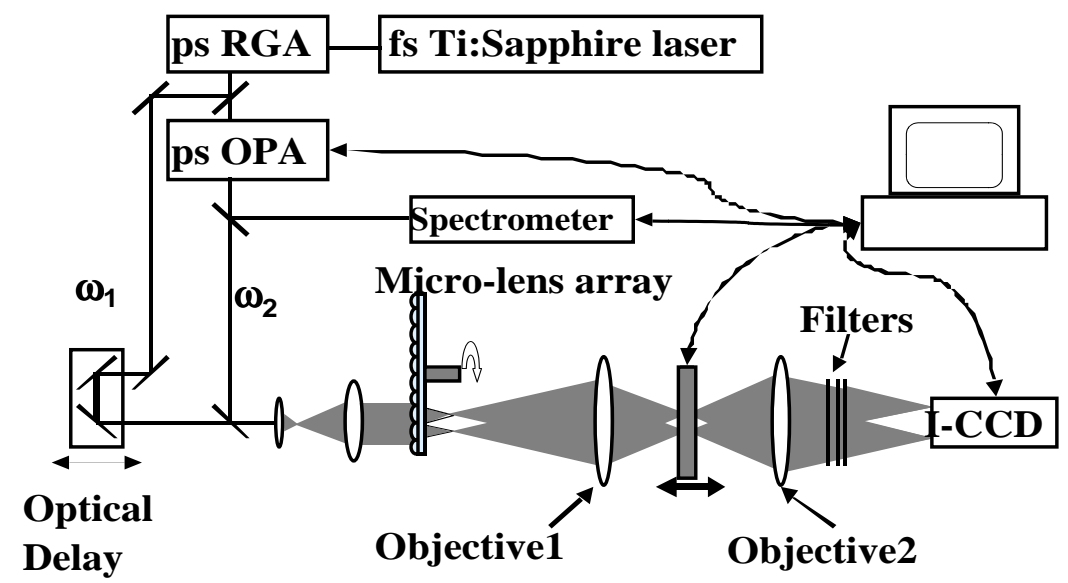

Figure 2. Schematic layout of multi-focus CARS microscopy system

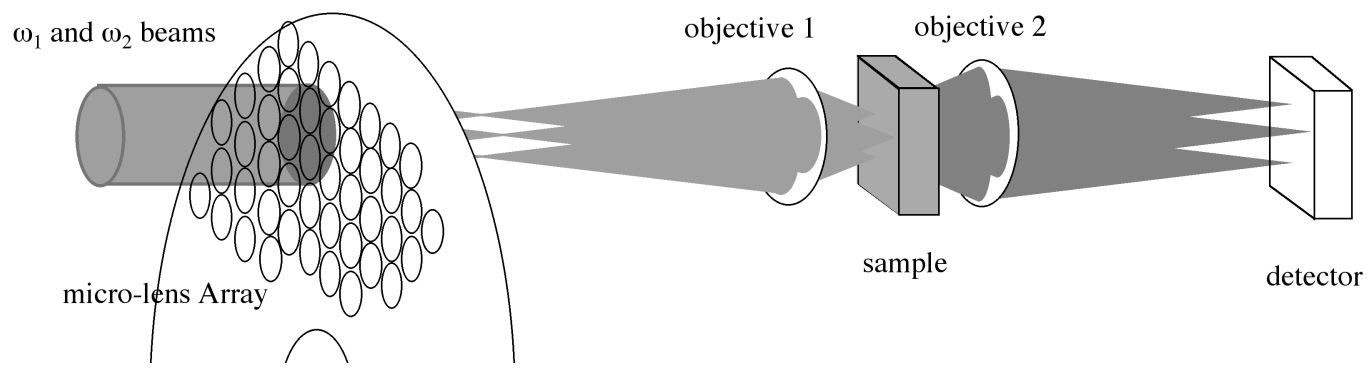

Figure 3. Principle of multi-focus CARS microscopy
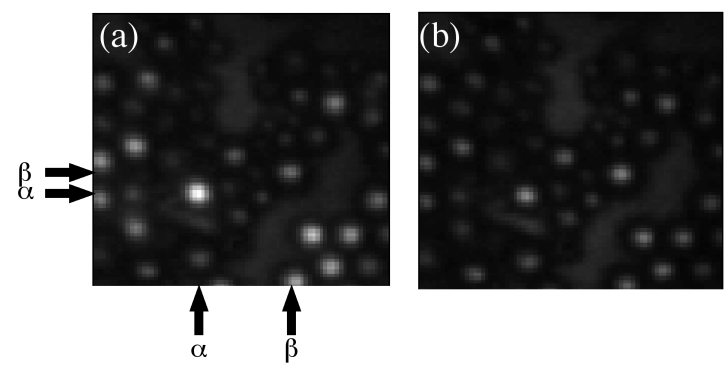

Figure 4: CARS image of the mixture of polystyrene at $990 \mathrm{~cm}^{-1}$ (a) and $1050 \mathrm{~cm}^{-1}(\mathrm{~b})$
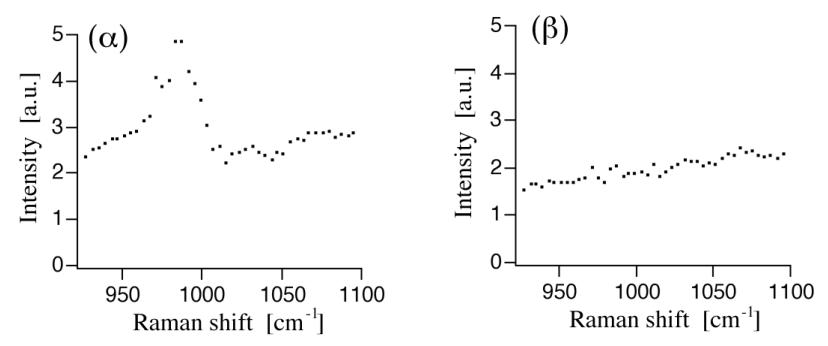

Figure 5: CARS spectra at $(\alpha)$ and $(\beta)$ in Fig. 4 